September 28, 1994

TAUP-2199-94

hep-th/9409175

\title{
Kähler spinning particles
}

\author{
Neil Marcus* \\ School of Physics and Astronomy \\ Raymond and Beverly Sackler Faculty of Exact Sciences \\ Tel-Aviv University \\ Ramat Aviv, Tel-Aviv 69978, ISRAEL.
}

\begin{abstract}
We construct the $U(N)$ spinning particle theories, which describe particles moving on Kähler spaces. These particles have the same relation to the $N=2$ string as usual spinning particles have to the NSR string. We find the restrictions on the target space of the theories coming from supersymmetry and from global anomalies. Finally, we show that the partition functions of the theories agree with what is expected from their spectra, unlike that of the $N=2$ string in which there is an anomalous dependence on the proper time.
\end{abstract}

\footnotetext{
${ }^{*}$ Work supported in part by the US-Israel Binational Science Foundation, the German-Israeli Foundation for Scientific Research and Development and the Israel Academy of Science.

E-Mail: NEIL@HALO.TAU.AC.IL
} 


\section{Introduction}

The "spinning particle" [1] describes a free Dirac particle, moving in some $D_{-}$ dimensional space. Historically, the particle action led to that of the NSR string. Conversely, one can obtain the spinning particle by dimensionally reducing the NSR string [2] or the heterotic string [3] to one dimension. The particle can be generalized to an $N$-spinning particle with a gauged $O(N)$ symmetry, which in four dimensions describes a spin $N / 2$ particle $[4,5]$. The string can also be generalized, to the $N=2[6]$ and $N=4$ [7] strings. However, the dimensional reduction of these extended string theories does not give the $O(N)$ spinning particles. The (ungauged) $O(2)$ particle can be obtained by the dimensional reduction of the NSR string, but the $N>2$ theories can not be derived from string theories.

In this paper we shall construct the $U(N)$ spinning particles, which have the same relation to the $N=2$ string as the $O(N)$ particles have to the NSR string. As with the $N=2$ string, these theories are not directly relevant to the real world, since they always have an even number of time coordinates. Our original motivation for studying them was that since particle theories are so much simpler than string theories, the $U(N)$ particles could provide us with an insight into some of the puzzles posed by $N=2$ strings. These include the Lorentz-invariance and supersymmetry of the string [8], and the conflict between loop calculations in the string and in the corresponding field-theories $[9,10]$. (For a review see [11].)

A separate motivation is that spinning particle theories are one-dimensional supergravity theories*, and since most supergravity theories turn out to be useful in one way or another, they are interesting to consider in their own right. Thus, truncations of the $U(1)$ and $U(2)$ theories of this paper have already been used to give a particle description of the open and closed B-twisted topological sigma models, respectively [14].

In this regard, it is useful to recall some results in the classification of supergravity theories: first, we should perhaps stress the difference between supergravity theories in three to eleven dimensions, and those in one and two dimensions. Supergravity theories in $D>2$ are related by dimensional reduction and truncation. The most beautifulalthough possibly the least useful - ones are the "larger" supergravities $(N>2$ in four dimensions). They are essentially unique, with their scalar fields living in various homogeneous spaces [15]. (References on supergravity theories in various dimensions can be

${ }^{*}$ This is not the most general definition of a particle theory. For example, one has the superparticle [12] which is invariant under target-space instead of world line supersymmetries, as well as hybrid theories [13] that are combinations of the two. We shall not consider such theories further. 
found in [16].) The smaller theories are more complicated, since matter supermultiplets can be coupled to them in various ways. Thus $N=1$ theories in three dimensions can be written with the scalar fields describing a sigma model on an arbitrary Riemann space [17]. The scalars of $N=1$ theories in four dimensions describe a Hodge manifold [18], while $N=2$ theories in four dimensions lead to a quaternionic sigma model [18]. The supergravity theories can exist on any spacetime, as long as it is a spin manifold.

Two-dimensional supergravity theories are not the dimensional reduction of those in three dimensions. While it is not necessary to do so, their main interpretation is as string theories, with the scalar fields interpreted as coordinates on a target space which is spacetime. Thus one might expect the larger $(N>4)$ supergravity theories to live on particular spacetimes. Even if these theories exist, they would be rather esoteric, and they have not been constructed. Classically $N=1,2$ and 4 strings live on Riemann [19], Kähler [7] and either hyperkähler or quaternionic [7] spaces $^{\dagger}$. (Recall that global $N=1$, 2 and 4 sigma models live on Riemann [20], Kähler [21] and hyperkähler [22] spaces, respectively.) In addition, one also has the various heterotic strings. This certainly is not a general classification of string theories - for example one can introduce torsions into the string - but it does give an overview of the basic types of string theories.

The two-dimensional theories can be reduced to one dimension, where they become particle theories. The reduction of the NSR string can be generalized to give the usual $O(N)$ spinning particles, which exist on Riemann spaces (sometimes on spin manifolds only). The $U(N)$ theories to be considered here can be derived from the $N=2$ string, and they can be defined only on Kähler target spaces. One can also compactify the $N=4$ string, which we expect to give $U S p(N)$ spinning particles living on hyperkähler or quaternionic spaces; however we shall not consider these theories further in this work.

The rest of this paper is organized as follows: In section two we give a brief summary of the known spinning particle actions, in order to compare them to the Kähler spinning particles. Most of this section is a restatement of results in [5] in our notation. In section three we construct the $U(N)$ theories. We discuss the restrictions on the target space of the theories coming from supersymmetry and, after introducing a Chern-Simons term, from anomalies. We then find the spectra of the theories, and discuss their spacetime conformal invariance. In section four we calculate the one-loop partition function of the particle, and see that it gives the result expected from its spectrum, unlike the corresponding calculation in the string. We end with some conclusions.

\footnotetext{
${ }^{\dagger}$ Of course demanding conformal invariance of the string theory restricts the spaces to be essentially Ricci flat, and phenomenological constraints may lead one to further restrict the theories, for example to Calabi-Yau spaces.
} 


\section{Summary of the $O(N)$ spinning particle}

The simplest particle action is simply that of an (unspinning) scalar particle with mass $m$ moving in a $D$-dimensional Minkowski space. It can be described by the action $[23]$ :

$$
\mathcal{L}=\frac{1}{2 e} \dot{X}^{M} \dot{X}^{N}+\frac{e}{2} m^{2}
$$

where $X^{M}$ is a map from the world line of the particle to the target space, and $e$ is an einbein on the world line. Canonical quantization shows that $X^{M}$ and $P^{N}=\dot{X}^{N} / e$ have the usual commutation relations of coordinates and momenta, and the equation of motion of the einbein gives the constraint $P^{2}=m^{2}$.

The spinning particle has a one-dimensional supersymmetry, which is made local by the introduction of a gravitino $\psi$. The supersymmetric partners of the $X^{M}$ 's are the spinors $\chi^{M}$ 's, and the action in the massless case is given by [1]:

$$
\mathcal{L}=\frac{1}{2 e} \dot{X}^{M} \dot{X}^{N}+\frac{i}{e} \dot{X}^{M} \psi \chi^{M}+\frac{i}{2} \chi^{M} \dot{\chi}^{M}
$$

Canonically quantizing (2), one sees that the $\chi^{M}$ 's become gamma matrices, so one is describing a Dirac particle*. The importance of the local supersymmetry of the action is seen from the fact that the constraint coming from the equation of motion of the gravitino is the massless Dirac equation.

This construction can be generalized to the $N=2$ case $[24,4,5]$, where one has two gravitini $\psi_{I}$ and $2 D$ spinors $\chi_{I}^{M}$. This theory has a gauged $S O(2)$ symmetry, and in four dimensions it describes the field equations of a Maxwell field. One can continue generalizing to the $N$-extended spinning particle [4, 25,5], with gravitini $\psi_{I}$ and spinors $\chi_{I}^{M}$ in the $N$ of a local $O(N)$. The lagrangian of the massless " $O(N)$ spinning particle" in a $D$-dimensional Riemann space is [5]:

$$
\begin{aligned}
\mathcal{L}=\frac{1}{2 e} G_{M N} & \left(\dot{X}^{M}+i \psi \cdot \chi^{M}\right)\left(\dot{X}^{N}+i \psi \cdot \chi^{N}\right)+\frac{i}{2} G_{M N} \chi_{I}^{M} \mathcal{D} \chi_{I}^{N} \\
& -\frac{e}{8} R_{M N P Q} \chi^{M} \cdot \chi^{N} \chi^{P} \cdot \chi^{Q}
\end{aligned}
$$

where the "dots" denote contractions over $O(N)$ indices. Here $\mathcal{D}$ is the covariantized time derivative, improved with a connection for the $O(N)$ group and the pullback of the Christoffel connection:

$$
\mathcal{D} \chi_{I}^{M} \equiv \dot{\chi}_{I}^{M}-i A_{I J} \chi_{J}^{M}+\Gamma_{P Q}^{M} \dot{X}^{P} \chi_{I}^{Q}
$$

\footnotetext{
*In the massive case, one needs to introduce an extra spinor which becomes $\gamma^{5}$ upon quantization [1].
} 
At this stage the metric $G_{M N}$ appears to be arbitrary, but one can see that for $N>2$ supersymmetry forces the theory to be in flat space. Using the Noether procedure, one finds the local supersymmetry transformations:

$$
\begin{array}{ll}
\delta e=-2 i \alpha \cdot \psi & \delta A_{I J}=0 \\
\delta \psi_{I}=\mathcal{D} \alpha_{I} & \\
\delta X^{M}=-i \alpha \cdot \chi^{M} & \delta \chi_{I}^{M}=\frac{1}{e}\left(\dot{X}^{M}+i \psi \cdot \chi^{M}\right) \alpha_{I}+i \Gamma_{P Q}^{M} \alpha \cdot \chi^{P} \chi_{I}^{Q} .
\end{array}
$$

Under these, the lagrangian is invariant up to 3-fermi terms (and total derivatives), but one is left with the 5 -fermion terms:

$$
\begin{aligned}
\delta \mathcal{L}= & \frac{i}{4} R_{M N P Q}\left(\alpha \cdot \psi \chi^{M} \cdot \chi^{N}-2 \psi \cdot \chi^{M} \alpha \cdot \chi^{N}\right) \chi^{P} \cdot \chi^{Q} \\
& +\frac{i e}{8} R_{M N P Q ; R} \chi^{M} \cdot \chi^{N} \chi^{P} \cdot \chi^{Q} \alpha \cdot \chi^{R} .
\end{aligned}
$$

For the Dirac particle $(N=1)$ these extra terms vanish due to the symmetries of the Riemann tensor, so the action is supersymmetric. (In fact, in this case both of the 4 -fermi terms in the lagrangian (3) vanish identically.) Similarly, for $N=2$ the 5 -fermion terms vanish using the symmetries of the Riemann tensor and the Bianchi identity [5]. However, for $N>2$ the lagrangian is supersymmetric only for a flat target space, with $R_{M N P Q}=0$, so the $O(N>2)$ spinning particle is relatively uninteresting.

Without going into further detail, the $O(N)$ spinning particle has the following properties:

\section{- Spectrum}

The " $O(0)$ " theory describes a scalar moving in any Riemann space. The " $O(1)$ " theory describes a Dirac spinor. This theory has a global anomaly unless the target space is a spin manifold $[26,27]$. The $O(2)$ theory describes an antisymmetric tensor field with $D / 2$ indices - a photon if $D=4$. The theory has a global anomaly in odd dimensions. The $O(N>2)$ theory can be written only in flat space. In four dimensions it describes a spin $N / 2$ particle $[4,5]$. In $D$ (even) dimensions, it describes a particle whose representation is described by the rectangular Young tableaux with $D / 2$ rows and $N / 2$ columns $[28,5]$, with half a column representing a spinor index.

\section{- Chern-Simons term}

In the case $N=2$, the gauge group of the theory is an $S O(2) \simeq U(1)$. Thus, one can add the term $\epsilon^{I J} A_{I J}=\operatorname{Tr} A$ to the lagrangian. This is the simplest example of a Chern-Simons term. Note that this term breaks the $O(2)$ of the theory to an $S O(2)$. With the addition of the Chern-Simons term with coefficient $q-D / 2$, the theory describes an antisymmetric

$q$-tensor field in any $D$-dimensional Riemann space. ( $D$ can now be arbitrary, but $q$ must 
be an integer to avoid the global anomaly [5].) Thus the $N=2$ theory can describe any antisymmetric tensor particle on any Riemann space.

\section{- Conformal invariance}

The massless $O(N)$ theory is invariant under target-space dilations. In fact the theory is even invariant under conformal transformations of the target-space [28,5], and all conformal representations in all dimensions can be obtained from the $O(N)$ theory [29]. In the $S O(2)$ theory conformal invariance is spoiled if the Chern-Simons term is added, and indeed the theory of a general antisymmetric tensor field in $D$ dimensions is not conformally invariant.

\section{- Supersymmetry algebra}

The supersymmetry algebra in the $O(N)$ theory closes into field-dependent diffeomorphisms, supersymmetry transformations and gauge transformations. If $N>1$, one has to use the fermion equations of motion, so the algebra closes only on shell.

Finally, note that the gauge field $A_{I J}$ does not transform under the supersymmetry transformations in (5). This means that if one's interest is in writing the most general onedimensional supergravity theory — rather than a particle theory - one is free to gauge any subgroup of the $O(N)$ symmetry, from the full $O(N)$ to the trivial identity group. This is unlike the case of the string or of supergravity in higher dimensions. If one does not gauge the full group, the theory will not describe a particle in an irreducible representation of the Lorentz (or conformal) group. For example, if one does not introduce the gauge field in the $O(2)$ theory, the theory describes all antisymmetric tensor fields simultaneously.

\section{The $U(N)$ spinning particle}

\subsection{Lagrangian}

We have argued that the $O(N)$ particles of the previous section are related to the NSR string. To carry out the dimensional reduction, one first fixes the Weyl, super-Weyl and Lorentz transformations, as well as the spatial diffeomorphisms of the string by the gauge choices $e_{\alpha}^{a}=\operatorname{diag}(e, 1)$ and $\psi_{1}=0$. Then one gets the (ungauged) $O(2)$ particle, which can be truncated to the usual spinning particle*. The $O(2)$ particle is not related to the $N=2$ string, as one might have expected from its $U(1)$ gauge symmetry. This follows simply by counting fields: In the reduction of the $N=2$ theory, there are twice as many spinors as scalars, as in the $O(2)$ particle, but there are 2 complex instead of 2

* One might have expected to have obtained the $O(2)$ gauge field from the $e_{0}^{1}$ component of the zweibein, but this drops out of the action. 
real gravitini.

Thus reducing the $N=2$ string to one dimension leads to a new family of particle theories. The $N=2$ string lives on a $d$-complex dimensional Kähler space $(D=2 d)$, so the coordinates $X^{M}$ are split into $X^{\mu}$ and their complex conjugates $X^{* \bar{\mu}}$. In analogy to the $S O(N)$ particle, we introduce an index $i$ which will transform under a local $U(N)$. Then the spinors become $\chi_{i}^{\mu}$ and $\chi^{* i \bar{\mu}}$, and the gravitini become $\psi_{i}$ and $\psi^{* i}$. The lagrangian can be found by reducing the $N=2$ string (in the $U(2)$ case with only a $U(1)$ gauging), or simply by using the Noether procedure:

$$
\begin{aligned}
\mathcal{L}=\frac{1}{e} G_{\mu \bar{\mu}} & \left(\dot{X}^{* \bar{\mu}}+i \psi \cdot \chi^{* \bar{\mu}}\right)\left(\dot{X}^{\mu}+i \psi^{*} \cdot \chi^{\mu}\right)+i G_{\mu \bar{\mu}} \chi^{* i \bar{\mu}} \mathcal{D} \chi_{i}^{\mu} \\
& -\frac{e}{2} R_{\mu \bar{\mu} \nu \bar{\nu}} \chi^{\mu} \cdot \chi^{* \bar{\mu}} \chi^{\nu} \cdot \chi^{* \bar{\nu}} .
\end{aligned}
$$

Here the derivative $\mathcal{D}$ is again covariantized with respect to diffeomorphisms and with respect to the local $U(N)$ :

$$
\mathcal{D} \chi_{i}^{\mu} \equiv \dot{\chi}_{i}^{\mu}-i A_{i}^{j} \chi_{j}^{\mu}+\Gamma_{\rho \sigma}^{\mu} \dot{X}^{\rho} \chi_{i}^{\sigma}
$$

Recall that in a Kähler space, the only nonvanishing components of the Christoffel symbol are $\Gamma_{\mu \nu}^{\rho}$ and its complex conjugate.

\subsection{Supersymmetry}

The supersymmetry transformations are given by

$$
\begin{aligned}
& \delta e=-i \alpha \cdot \psi^{*}-i \alpha^{*} \cdot \psi \\
& \delta \psi_{i}=\mathcal{D} \alpha_{i} \\
& \delta A_{i}{ }^{j}=0 \\
& \delta X^{* \bar{\mu}}=-i \alpha \cdot \chi^{* \bar{\mu}} \\
& \delta \chi_{i \bar{\mu}}=\frac{1}{e} G_{\mu \bar{\mu}}\left(\dot{X}^{\mu}+i \psi^{*} \cdot \chi^{\mu}\right) \alpha_{i}-i \Gamma_{\bar{\mu} \bar{\rho}, \sigma} \alpha \cdot \chi^{* \bar{\rho}} \chi_{i}^{\sigma} .
\end{aligned}
$$

We have chosen to write the transformation of $\chi_{i \bar{\mu}}$ with its spacetime index lowered, since the transformation of $\chi_{i}^{\mu}$ involves both $\alpha$ and $\alpha^{*}$. As in the $O(N)$ case, one finds 5 -fermi terms in the supersymmetry variation of the lagrangian:

$$
\begin{aligned}
\delta \mathcal{L}= & \frac{i}{2} R_{\mu \bar{\mu} \nu \bar{\nu}}\left(\alpha \cdot \psi^{*} \chi^{\mu} \cdot \chi^{* \bar{\mu}}-2 \psi^{*} \cdot \chi^{\mu} \alpha \cdot \chi^{* \bar{\mu}}\right) \chi^{\nu} \cdot \chi^{* \bar{\nu}} \\
& +\frac{i e}{2} R_{\mu \bar{\mu} \nu \bar{\nu} ; \bar{\rho}} \chi^{\mu} \cdot \chi^{* \bar{\mu}} \chi^{\nu} \cdot \chi^{* \bar{\nu}} \alpha \cdot \chi^{* \bar{\rho}}+\text { h.c. }
\end{aligned}
$$


In the $U(1)$ theory the curvature terms vanish both here and in the lagrangian (7), since in a Kähler space $R_{\mu \bar{\mu} \nu \bar{\nu}}$ is symmetric in each set of indices. In the $U(2)$ theory the extra terms in (10) vanish because of the symmetries of $R_{\mu \bar{\mu} \nu \bar{\nu}}$ and because of the Bianchi identity, which in a Kähler space states that $R_{\mu \bar{\mu} \nu \bar{\nu} ; \bar{\rho}}$ is totally symmetric in the "barred" indices. Thus the $U(1)$ and $U(2)$ theories can be written in any Kähler space. As in the $O(N)$ case, the $U(N>2)$ theories are supersymmetric only in flat space, and are again of limited interest.

Note that since $A_{i}{ }^{j}$ does not transform under supersymmetry transformations, one can again restrict the gauging of the theory to any subgroup of the $U(N)$ symmetry. For example, the open and closed $B$-particles of ref. [14] are given by the $U(1)$ and $U(2)$ lagrangians of (7), with no gauging whatsoever and with the $\psi_{i}$ 's set to zero. (Thus in these theories only the $\alpha^{* i}$ supersymmetry transformations are local; those of the $\alpha_{i}$ 's are not.)

Finally, upon commuting two supersymmetry transformations with parameters $\alpha_{i}$ and $\beta_{j}$, one finds a diffeomorphism by $\xi \equiv i / e\left(\alpha^{*} \cdot \beta+\alpha \cdot \beta^{*}\right)$, a supersymmetry transformation with parameter $-\xi \psi_{i}$ and a $U(N)$ transformation with parameter $-\xi A_{i}{ }^{j}$. For $N>1$, the algebra closes only with the use of the equation of motion of the $\chi_{i}^{\mu}$, s.

\subsection{Chern-Simons term, the spectrum, and anomalies.}

The spectrum of the particle can be changed by adding the Chern-Simons term

$$
\mathcal{L}_{C S}=\left(\frac{d}{2}-q\right) A_{i}^{i}
$$

to the lagrangian of the particle, where for now $q$ is an arbitrary parameter. Note that the Chern-Simons term exists for all $N$, and that it is consistent with supersymmetry, since $A_{i}{ }^{j}$ is totally invariant.

In canonically quantizing the theory, the $X^{\mu}$ s and their conjugates $P_{\mu}$ become position and momentum operators, as do $X^{* \bar{\mu}}$ and $P_{\bar{\mu}}^{*}$. The $\chi^{* i \bar{\mu}}$, s can be taken to be creation operators and the $\chi_{i}^{\mu}$ 's to be annihilation operators, so the general state with momentum $P_{\mu}$ is built from the vacuum state $\left|P_{\mu}\right\rangle$ by applying some number of $\chi^{* i \bar{\mu}}$ 's. As usual, the equations of motion of the supergravity fields $e, \psi_{i}$ and $A_{i}{ }^{j}$ constrain the Hilbert space: Thus, varying $A_{i}^{j}$ in the combined lagrangian of (7) and (11) gives:

$$
G_{\mu \bar{\mu}} \chi^{* i \bar{\mu}} \chi_{j}^{\mu}=q \delta_{j}^{i}
$$

where we have used a normal ordering scheme that is symmetric between the $\chi$ 's and $\chi^{*}$ 's. Acting the " $i i$ " element of this constraint on a state $\Psi$ tells us that there must be 
exactly $q \chi^{* i}$ 's in the state for each $i$, so $\Psi$ has the form:

$$
\Psi=F_{\bar{\mu}_{1} \cdots \bar{\mu}_{q} \bar{\nu}_{1} \cdots \bar{\nu}_{q} \cdots} \chi^{* 1 \bar{\mu}_{1}} \cdots \chi^{* 1 \bar{\mu}_{q}} \chi^{* 2 \bar{\nu}_{1}} \cdots \chi^{* 2 \bar{\nu}_{q}} \ldots\left|P_{\mu}\right\rangle
$$

This means that the theory is empty unless $q$ is an integer between 0 and $d$. (Thus the Chern-Simons term is necessary in an odd number of complex dimensions). The lack of a spectrum when $q$ is not an integer is an indication of the global anomaly of the theory in that case [30].

The off-diagonal elements of the constraint (12) impose a symmetry between the $i$ and $j$ indices of the tensor $F$, implying that it is represented by the rectangular Young tableaux

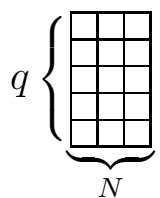

of $S U(d)$. (This is similar to the case of the $O(N)$ particle, where the state has $D / 2$ rows and $N / 2$ columns.) In general, the holonomy group of the Kähler space will be a full $U(d)$. Again using a symmetric normal ordering, and normalizing the $U(1)$ charge of $\chi_{i}^{\mu}$ to be 1 , one sees that the particle represented by $\Psi$ has charge $N(d / 2-q)$. If $N d$ is odd the particle will have a half-integral $U(1)$ charge, indicating that it is spinor-like. (The simplest case with $N=1$ gives the various pieces of the $S O(D)$ spinors broken into $U(d)$ representations, as $q$ is varied.) In these cases the theory again has a global anomaly unless the space supports a spin structure. In a Calabi-Yau space this problem never arises, since the holonomy group of the spacetime is $S U(d)$.

The equations of motion of the state $\Psi$ are given by varying the lagrangian with respect to the gravitini and the einbein. In order to avoid normal ordering problems we shall for simplicity restrict ourselves here to the case of flat space ${ }^{\dagger}$. The einbein constraint shows that the particle is massless: $G^{\mu \bar{\mu}} P_{\mu} P_{\bar{\mu}}^{*}=0$, while the constraints from the gravitini give the equations of motion

$$
\begin{aligned}
& P^{\bar{\mu}_{1}} F_{\bar{\mu}_{1} \cdots \bar{\mu}_{q} \bar{\nu}_{1} \cdots \bar{\nu}_{q} \cdots}=0 \\
& P_{[\bar{\mu}}^{*} F_{\left.\bar{\mu}_{1} \cdots \bar{\mu}_{q}\right] \bar{\nu}_{1} \cdots \bar{\nu}_{q} \cdots}=0 .
\end{aligned}
$$

Eqs. (15) are analogous to the equation of motion and Bianchi identity of the photon (which one can get from the $O(2)$ theory): $\partial_{M} F_{M N}=0$ and $\partial_{[L} F_{M N]}=0$. By going into a light-cone frame, one can see that the field strength in (14) is reduced on shell to

\footnotetext{
${ }^{\dagger}$ Such problems lead to an unusual choice of creation and annihilation operators in the B-particle [14].
} 
a connection in the

$$
q-1\{\underbrace{\sqrt{+1}}_{N}
$$

representation of the massless little group $U(d-2)$. Note that the theory has no propagating particles for $q=0$ and $q=d$.

\subsection{Conformal invariance}

In addition to the worldline symmetries we have discussed, the $U(N)$ particle is covariant under holomorphic diffeomorphisms. It is therefore invariant under holomorphic isometries of the spacetime. For example, in flat space it is invariant under $U(d)$ "Lorentz" transformations. The bosonic $(N=0)$ string is invariant under the more general class of conformal transformations, defined by $\delta X^{\mu}=\xi^{\mu}$, with $\xi^{\mu}$ restricted to be a conformal Killing vector:

$$
\begin{aligned}
& \xi_{\bar{\mu}, \mu}+\bar{\xi}_{\mu, \bar{\mu}}=2 G_{\mu \bar{\mu}} \rho \\
& \xi_{\bar{\mu} ; \bar{\nu}}+\xi_{\bar{\nu} ; \bar{\mu}}=0
\end{aligned}
$$

here the "scale-factor" $\rho$ defined by (17a) vanishes if $\xi^{\mu}$ is a Killing vector, in which case one is back to an isometry transformation of the spacetime. If one attempts to generalize the conformal symmetry to the $U(N)$ lagrangian, one finds that a term

$$
\delta \mathcal{L}=\frac{i}{e} \xi_{, \bar{\nu}}^{\mu} \dot{X}^{* \bar{\nu}} \psi \cdot \chi_{\mu}^{*}+\text { h.c. }
$$

can not be canceled. This means that $(17 \mathrm{~b})$ must be replaced by the stronger condition that $\xi^{\mu}$ be a holomorphic conformal Killing vector, with

$$
\xi_{, \bar{\nu}}^{\mu}=0
$$

In addition, one finds the unwanted terms

$$
\delta \mathcal{L}=i \dot{X}^{* \bar{\mu}} \chi^{* \bar{\nu}} \cdot \chi^{\mu}\left(-\rho_{, \bar{\mu}} G_{\mu \bar{\nu}}+\rho_{, \bar{\nu}} G_{\mu \bar{\mu}}\right)+\text { h.c. }
$$

These terms vanish identically when $d=1$, but otherwise one must impose the condition that $\rho$ be constant. Aside from holomorphic isometries of the spacetime, this leaves only constant dilations. By going to Riemann-normal coordinates one can see that such holomorphic dilations are only possible in flat space. In this case there is clearly a dilation symmetry, with the fields $X^{\mu}, e, \chi_{i}^{\mu}, \psi_{i}$ and $A_{i}{ }^{j}$ having weights $1,2,0,1$ and 0 , respectively. 
We thus see that there are only interesting conformal transformations when the spacetime is a Riemann surface $(d=1)$. (Note, however, that Riemann surfaces can not support massless propagating particles, since they can not have both space and time coordinates, so the theory is basically topological in this case.) Then one does have invariance under the full conformal group, with the transformations:

$$
\begin{aligned}
& \delta X^{\mu}=\xi^{\mu} \\
& \delta \chi_{i}^{\mu}=\xi^{\mu}{ }_{, \nu} \chi_{i}^{\nu}-\rho \chi_{i}^{\mu} \\
& \delta e=2 \rho e \\
& \delta \psi_{i}=\rho \psi_{i}+e \rho_{, \mu} \chi_{i}^{\mu} \\
& \delta A_{i}{ }^{j}=\frac{1}{N+1}\left(\delta_{i}^{j} \delta_{l}^{k}-N \delta_{i}^{k} \delta_{l}^{j}\right)\left(\rho_{, \mu} \psi^{* l} \chi_{k}^{\mu}-\rho_{, \bar{\mu}} \psi_{k} \chi^{* l \bar{\mu}}-e \rho_{, \mu \bar{\mu}} \chi^{* l \bar{\mu}} \chi_{k}^{\mu}\right) .
\end{aligned}
$$

The transformation of $A_{i}{ }^{j}$ can actually be written as various linear combinations of the two sets of terms in (18); we have chosen the combination that leaves $\operatorname{Tr} A$ invariant, so the Chern-Simons lagrangian (11) does not break the conformal symmetry.

\section{The partition function}

Thus far, we have found the spectrum of the particle using Hamiltonian quantization. It would be nice to also be able to calculate amplitudes of the theory. However, since the particle lagrangian describes a free particle, the only quantity one can calculate without introducing interactions is the one-loop partition function. This is nevertheless interesting, since the analogous partition function of the $N=2$ string disagrees with the result expected from its spectrum. Recall that the partition function of a particle with mass $m$ moving in a Euclidean $D$-dimensional space is [31]:

$$
\begin{aligned}
\mathcal{F} & =-\frac{1}{2} \operatorname{Tr} \log \left(p^{2}+m^{2}\right) \\
& =\frac{V}{2} \int_{0}^{\infty} \frac{\mathrm{d} T}{T^{1+D / 2}} e^{-m^{2} T},
\end{aligned}
$$

where $T$ is the Schwinger proper-time parameter. The $N=2$ string describes only massless degrees of freedom in a flat 2-complex dimensional space $(d=2, D=4)$. However, its partition function is [9]

$$
\mathcal{F}=\frac{1}{2} \int_{\mathcal{M}} \frac{\mathrm{d}^{2} \tau}{\tau_{2}^{2}},
$$

which is compatible with that of the particle only if $D=2$ ! There is a similar disagreement between the one-loop three-point function in the string [10] and that of the "Plebanski" 
field theory [32] which should be the effective field theory describing the string. One of the explanations advocated to solve this problem [32] is that the complex nature of the Kähler target space of the $N=2$ string means that one should use a complex Schwinger parameter. The problem could also come from the $U(1)$ gauge field in the theory, or have an intrinsically stringy nature. Since the $U(N)$ particle is intimately related to the $N=2$ string, and shares many of its features, calculating its partition function can distinguish between the various possibilities.

We shall start with the $U(1)$ theory in flat space with complex dimension $d$, on a worldline which is a circle with proper length $T$. We need to evaluate the path integral over the lagrangian (7) with the Chern-Simons term (11). The new feature of the $U(N)$ string is that the gauge field $A$ can not be completely gauged away, since it can have a nontrivial Wilson line around the circle. The allowed gauge choice is $A=\theta / T$, with $\theta$ ranging from 0 to $2 \pi$. This means that the spinors $\chi^{\mu}$ and the gravitino $\psi$ pick up a phase of $\theta$, when going around the circle. Most of the path integral is standard: As usual $[31,23]$, the integral over the einbein (gauged to $e=T$ ) modulo diffeomorphisms gives

$$
\frac{1}{2} \int_{0}^{\infty} \frac{\mathrm{d} T}{T},
$$

and the path integral over the $X^{\mu}$ 's gives

$$
V T^{d} \operatorname{det}^{\prime}\left(-\partial_{t}^{2}\right)^{-d}=\frac{V}{T^{d}} .
$$

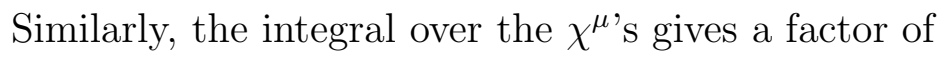

$$
\left(\operatorname{det}_{\theta} i \partial_{t}\right)^{d} \text {, }
$$

where the index on the "det" is to remind us of the shifted boundary conditions of the fermions. For a generic $A$, the gravitino can be completely gauged away using the supersymmetry transformation, with the resulting Jacobian reducing the exponent in (23) to $d-2$.

The interesting question, of course, is what comes from the integral over $A$, modulo the $U(1)$ transformations. The zero-mode integration gives $\int_{0}^{2 \pi} \mathrm{d} \theta / \sqrt{T}$; the integration over constant gauge transformations gives a factor of $2 \pi \sqrt{T}$ in the denominator, and the Jacobian is $\operatorname{det}^{\prime} i \partial_{t}=T$. All together, this means that the $A$ integration simply gives rise to an insertion of the projection operator $\int_{0}^{2 \pi} \frac{\mathrm{d} \theta}{2 \pi}$. Since the gauge-field integration contributes no factors of $T$, there will be no anomalous powers of $T$ in the particle.

Putting together the above results, and adding the Chern-Simons term (11), we find

$$
\mathcal{F}=\frac{V}{2} \int_{0}^{\infty} \frac{\mathrm{d} T}{T^{1+d}} \int_{0}^{2 \pi} \frac{\mathrm{d} \theta}{2 \pi} e^{i(q-d / 2) \theta}(2 \sin \theta / 2)^{d-2},
$$


where we have used the result that (up to an ill-defined $\operatorname{sign}$ ) $\operatorname{det}_{\theta} i \partial_{t}=2 \sin (\theta / 2$ ). Evaluating the $\theta$ integral, and comparing (24) to (19), one sees that the $U(1)$ particle

describes $\left(\begin{array}{c}d-2 \\ q-1\end{array}\right)$ massless particles in a $d$-complex dimensional space. This is exactly the number of degrees of freedom that one expects from the Hamiltonian calculation: the field strength of the particle is described by an antisymmetric tensor with $q$ indices (14); the equations of motion (15) reduce this to a light-cone connection with $q-1$ indices (16).

To check whether or not subtleties occur when the gauge group is nonabelian, we shall also carry out the calculation of the partition function in the $U(2)$ theory. (The combinatorics of the $U(N)$ case will be left to our more intrepid readers.) The integrals over $e$ and $X$ are the same as before. In this case $A_{i}{ }^{j}$ can be gauge fixed to $A=\operatorname{diag}(\theta, \phi)$, where both $\theta$ and $\phi$ go from 0 to $2 \pi$. The fermionic integrations are the same as in the $U(1)$ result (23), duplicated over $\theta$ and $\phi$. Similarly, the integrations over the diagonal zero modes of $A$ give projection operators for both $\theta$ and $\phi$. The only new features are that there is a factor of $1 / 2$, since a $90^{\circ}$ rotation in the $y-z$ plane interchanges $\theta$ and $\phi$, and that the two off-diagonal gauge transformations each contribute the Jacobian $\operatorname{det}_{\theta-\phi} i \partial_{t}$. The integrations over $\theta$ and $\phi$ then give a factor of

$$
\begin{aligned}
& -\frac{1}{2} \int_{0}^{2 \pi} \frac{\mathrm{d} \theta}{2 \pi} \int_{0}^{2 \pi} \frac{\mathrm{d} \phi}{2 \pi} e^{i(q-d / 2)(\theta+\phi)}(2 \sin (\theta / 2-\phi / 2))^{2}(2 \sin \theta / 2)^{d-2}(2 \sin \phi / 2)^{d-2} \\
& \quad=\frac{(d-1) !(d-2) !}{(d-q) !(d-q-1) ! q !(q-1) !} .
\end{aligned}
$$

This is the dimension of the $U(d-2)$ representation of the particle in (16), for $N=2$, so the path integral calculation of the partition function is again in perfect agreement with the result expected from the canonical quantization of the particle. There is no sign of the anomalies of the $N=2$ string.

\section{Conclusions}

We have constructed the $U(N)$ spinning particles - one dimensional supergravity theories with $N$ complex local supersymmetries on the world line and a local $U(N)$ invariance. These theories describe massless particles moving in a Kähler spacetime with complex dimension $d$. The $U(1)$ and $U(2)$ theories can be defined on any Kähler space; consistency with supersymmetry forces the $U(N>2)$ theories to be in flat space. The theories have a spacetime conformal invariance only if the target space is a Riemann surface $(d=1)$.

The spectrum of the theories depends on the coefficient of the Chern-Simons term $(d / 2-q) \operatorname{Tr} A$, which can be added to the lagrangian for any $N$. To avoid global anomalies 
$q$ must be an integer, and the manifold must support a spin structure if $N d$ is odd. The constraint coming from the gauge field implies that the "Lorentz group" $U(d)$ representation of the field strength of the particle is given by a rectangular Young tableaux with $q$ rows and $N$ columns. The equations of motion (15) then show that the particle is in the representation of the little group $U(d-2)$ with $q-1$ rows and $N$ columns.

The $U(N)$ particle can be regarded as a toy model for the $N=2$ string, to which it is intimately related. (The $U(1)$ and $U(2)$ theories can be obtained by dimensionally reducing the $N=2$ string, and then gauging the global symmetry of the theory.) One result that has implications for the string is that the partition function of the particle, calculated by performing the path integral on the circle with a flat target space, is in perfect agreement with what one would expect from the spectrum of the particle. This is in contradistinction to the string, in which modular invariance forces the string partition function to have a peculiar dependence on the proper time $\tau$. The fact that this does not occur in the particle rules out several of the explanations proposed for this phenomenon.

Other unsettled issues in the $N=2$ string are whether or not it is the same as the $N=4$ string, and whether or not it has spacetime supersymmetry and a full $S O(D)$ Lorentz invariance [8]. It is less clear here what implications can be drawn from the particle. One would not expect to be able to see spacetime supersymmetry in any (non super) particle. The general $U(N)$ particle defined on some Kähler space certainly does not have an $S O(D)$ Lorentz invariance. Indeed, the spectrum will not even fall into representations of $S O(D)$. It is also not equivalent to a $U S p(N / 2)$ particle. However, these properties may still hold in the special case of the $N=2$ string, for which spacetime must be hyperkähler in four real dimensions.

Some issues which we have not explored, but which should not pose any great difficulty, are how to add masses to the $U(N)$ theories, and how to construct the $U S p(N)$ theories, which are related to the $N=4$ strings. Masses have been included in the original spinning particle [1], and in the $O(N)$ particle [4], although only in flat space. One should be able to include them in the $U(N)$ strings in the same way. Alternatively, one can introduce the masses by dimensionally reducing the $N=2$ string using the ScherkSchwarz mechanism [33]. Also, following the line of this paper, it should not be hard to find the proposed $U S p(N)$ particles, for example by dimensionally reducing the $N=4$ string [7]. As with that string, these theories should be definable only on hyperkähler or quaternionic spacetimes. A more difficult and more interesting problem is to classify all spinning particle theories. 


\section{References}

[1] F.A. Berezin and M.S. Marinov, Ann. Physics 104 (1977) 336;

L. Brink, S. Deser, B. Zumino, P. Di Vecchia and P. Howe, Phys. Lett. 64B (1976) 435.

[2] L. Brink, P. Di Vecchia and P. Howe, Phys. Lett. 65B (1976) 471;

S. Deser and B. Zumino, ibid. 369.

[3] D.J. Gross, J.A. Harvey, E. Martinec and R. Rohm, Nucl. Phys. B256 (1985) 253.

[4] V.D. Gershun and V.I. Tkach, Pis'ma Zh. Exp. Theor. Fiz. 29 (1979) 320 [Sov. Phys. JETP 29 (1979) 288].

[5] P.S. Howe, S. Penati, M. Pernici and P. Townsend, Phys. Lett. 215B (1988) 555, Class. Quantum Grav. 6 (1989) 1125.

[6] L. Brink and J.H. Schwarz, Nucl. Phys. B121 (1977) 285.

[7] M. Pernici and P. van Nieuwenhuizen, Phys. Lett. 169B (1986) 381.

[8] W. Siegel, Phys. Rev. Lett. 69 (1992) 1493, hep-th/9207043; Phys. Rev. D46 (1992) 3235, hep-th/9205075; Phys. Rev. D47 (1993) 2504, hep-th/9207043.

[9] S.D. Mathur and S. Mukhi, Nucl. Phys. B302 (1988) 130.

[10] M. Bonini, E. Gava and R. Iengo, Mod. Phys. Lett. A6 (1991) 795.

[11] N. Marcus, "A tour through N=2 strings", in "String theory, quantum gravity and the unification of the fundamental interactions", eds. M. Bianchi, F. Fucito, E. Marinari and A. Sagnotti (World Scientific 1993), hep-th/9207024.

[12] R. Casalbuoni, Phys. Lett. 62B (1976) 49;

L. Brink and J.H. Schwarz, Phys. Lett. 100B (1981) 310.

[13] E.A. Bergshoeff and J.W. van Holten, Phys. Lett. 226B (1989) 93.

[14] N. Marcus and S. Yankielowicz, "The topological B model as a twisted spinning particle", Tel-Aviv preprint TAUP-2192-94, hep-th/9408116, to appear in Nuclear Physics B.

[15] J.H. Schwarz, Phys. Lett. 95B (1980) 219;

E. Cremmer, "Supergravities in 5 dimensions", in "Superspace and supergravity", ed. S.W. Hawking and M. Roček (Cambridge Univ. Press, 1981);

B. Julia, "Group disintegrations", ibid.

[16] "Supergravities in diverse dimensions", ed. A. Salam and E. Sezgin (World Scientific, 1989), 2 volumes. 
[17] B. de Wit, A.K. Tollsten and H. Nicolai, hep-th/9208074, Nucl. Phys. B392 (1993) 3.

[18] J. Bagger and E. Witten, Phys. Lett. 115B (1982) 202, Nucl. Phys. B222 (1983) 1.

[19] E. Bergshoeff, S. Randjbar-Daemi, A. Salam, H. Sarmadi and E. Sezgin, Nucl. Phys. B269 (1986) 77.

[20] D.Z. Freedman and P.K. Townsend, Nucl. Phys. B177 (1981) 282.

[21] B. Zumino, Phys. Lett. 87B (1979) 203.

[22] L. Álvarez-Gaumé and D.Z. Freedman, Comm. Math. Phys. 80 (1981) 443.

[23] See, for example, A.M. Polyakov, "Gauge fields and strings" (Harwood Academic, 1987).

[24] L. Brink, P. Di Vecchia and P. Howe, Nucl. Phys. B118 (1977) 76.

[25] M. Henneaux and C. Teitelboim, "First and second quantized point particles of any spin", in "Quantum mechanics of fundamental systems 2", ed. C. Teitelboim and J. Zanelli (Plenum Press, 1989).

[26] E. Witten, "Global anomalies in string theory", in "Symposium on Anomalies, Geometry and Topology", ed. W.A. Bardeen and A.R. White (World Scientific, 1985).

[27] L. Álvarez-Gaumé, Comm. Math. Phys. 90 (1983) 161.

[28] W. Siegel, Int. J. Mod. Phys. A3 (1988) 2713.

[29] W. Siegel, Int. J. Mod. Phys. A4 (1989) 2015.

[30] S. Elitzur, Y. Frishman, E. Rabinovici and A. Schwimmer, Nucl. Phys. B273 (1986) 93.

[31] J. Polchinski, Comm. Math. Phys. 104 (1986) 37.

[32] H. Ooguri and C. Vafa, Nucl. Phys. B361 (1991) 469.

[33] J. Scherk and J.H. Schwarz, Nucl. Phys. B153 (1979) 61. 